\title{
DARK MATTER CAUSTICS
}

\author{
P. SIKIVIE, W. KINNEY \\ Department of Physics, University of Florida, Gainesville, \\ FL 32611, USA \\ E-mail: sikivie@phys.ufl.edu,kinney@phys.ufl.edu
}

\begin{abstract}
The late infall of cold dark matter onto an isolated galaxy such as our own produces flows with definite velocity vectors at any physical point in the galactic halo. It also produces caustics which are places where the dark matter density is very large. The outer caustics are topological spheres whereas the inner caustics are rings. The self-similar model of galactic halo formation predicts that the caustic ring radii $a_{n}$ follow the approximate law $a_{n} \sim 1 / n$. In a recent study of 32 extended and well-measured galactic rotation curves, we found evidence for this law.
\end{abstract}

\section{Introduction}

Before the onset of galaxy formation but after the time $t_{e q}$ of equality between matter and radiation, the velocity dispersion of the cold dark matter candidates is very small, of order $\delta v_{a}(t) \sim 3 \cdot 10^{-17}\left(\frac{10^{-5} \mathrm{eV}}{m_{a}}\right) \quad\left(\frac{t_{0}}{t}\right)^{2 / 3}$ for axions and $\delta v_{W}(t) \sim 10^{-11}\left(\frac{G e V}{m_{W}}\right)^{1 / 2}\left(\frac{t_{0}}{t}\right)^{2 / 3}$ for WIMPs, where $t_{0}$ is the present age of the universe and $m_{a}$ and $m_{W}$ are respectively the masses of the axion and the WIMP. In the context of galaxy formation, such small velocity dispersions are entirely negligible. Massive neutrinos, on the other hand, have primordial velocity dispersion $\delta v_{\nu}(t) \simeq 5.310^{-4}\left(\frac{e V}{m_{\nu}}\right) \quad\left(\frac{t_{0}}{t}\right)^{2 / 3}$ which is comparable to the virial velocity in galaxies and therefore non-negligible in the context of galaxy formation $\mathrm{t}$. This is the reason why massive neutrinos are called 'hot dark matter'.

Collisionless dark matter particles lie on a thin 3-dimensional (3D) sheet in $6 \mathrm{D}$ phase-space. The thickness of this sheet is the primordial velocity dispersion $\delta v$. If each of the aforementioned species of collisionless particles is present, the phase-space sheet has three layers, a very thin layer of axions, a medium layer of WIMPs and a thick layer of neutrinos. The phase-space sheet is located on the 3D hypersurface of points $(\vec{r}, \vec{v}): \vec{v}=H(t) \vec{r}+\Delta \vec{v}(\vec{r}, t)$ where $H(t)=\frac{2}{3 t}$ is the Hubble expansion rate and $\Delta \vec{v}(\vec{r}, t)$ is the peculiar velocity field. Fig. 1 shows a $2 \mathrm{D}$ section of $6 \mathrm{D}$ phase-space along the $(z, \dot{z})$ plane. The wiggly line is the intersection of the $3 \mathrm{D}$ sheet on which the particles lie in phase-space with the plane of the figure. The thickness of the line is the velocity dispersion $\delta v$, whereas the amplitude of the wiggles in the line is the peculiar velocity $\Delta v$. If there were no peculiar velocities, the line would be 


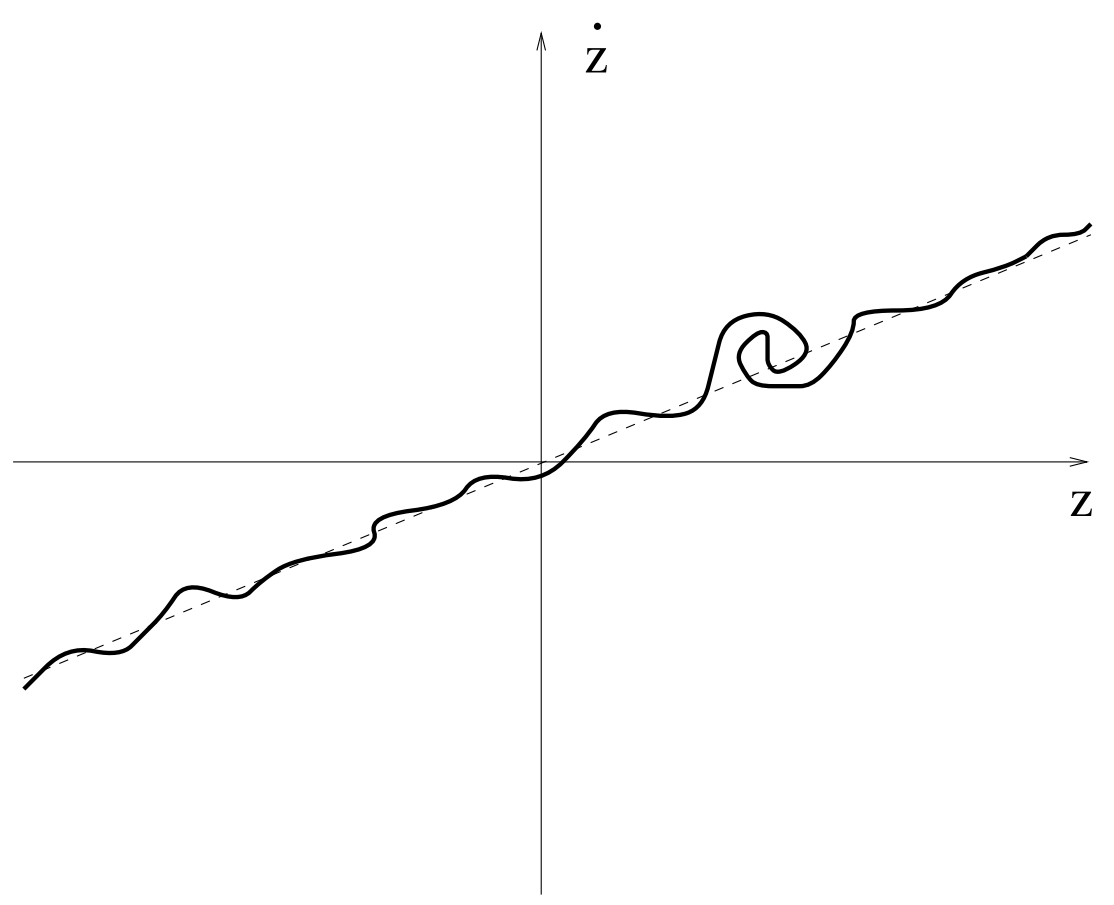

Figure 1: The wiggly line represents the intersection of the $(z, \dot{z})$ plane with the 3D sheet on which the collisionless dark matter particles lie in phase-space. The thickness of the line is the primordial velocity dispersion. The amplitude of the wiggles in the $\dot{z}$ direction is the velocity dispersion associated with density perturbations. Where an overdensity grows in the non-linear regime, the line winds up in clockwise fashion. One such overdensity is shown.

straight since $\dot{z}=H(t) z$ in that case.

The peculiar velocities are associated with density perturbations and grow by gravitational instability as $\Delta v \sim t^{2 / 3}$. On the other hand the primordial velocity dispersion decreases on average as $\delta v \sim t^{-2 / 3}$, consistently with Liouville's theorem. When an overdensity enters the non-linear regime, the particles in its vicinity fall back onto it. This implies that the phase-space sheet 'winds up' there in clockwise fashion. One such overdensity is shown in Fig. 1. In the linear regime, there is only one value of velocity, i.e. one single flow, at a typical location in physical space, because the phase-space sheet covers physical space only once. On the other hand, inside an overdensity in the non-linear regime, the phase-space sheet covers physical space multiple times implying that there are several (but always an odd number of) flows at such 
locations.

At the boundary surface between two regions one of which has $n$ flows and the other $n+2$ flows, the physical space density is very large because the phase-space sheet has a fold there. At the fold, the phase-space sheet is tangent to velocity space and hence, in the limit of zero velocity dispersion $(\delta v=0)$, the density diverges since it is the integral of the phase-space density over velocity space. The structure associated with such a phase-space fold is called a 'caustic'. It is a surface in physical space. It is easy to show that, in the limit of zero velocity dispersion, the density diverges as $d \sim \frac{1}{\sqrt{\sigma}}$ when the caustic is approached from the side with $n+2$ flows, where $\sigma$ is the distance to the caustic. Velocity dispersion cuts off the divergence.

As mentioned above, the process of galactic halo formation involves the local winding up of the phase-space sheet of collisionless dark matter particles. If the galactic center is approached from an arbitrary direction at a given time, the local number of flows increases. First, there is one flow, then three flows, then five, seven... The number of flows at our location in the Milky Way galaxy today has been estimated 10 to be of order 100 . The boundary between the region with one (three, five, ...) and the region with three (five, seven, ...) flows is the location of a caustic which is topologically a sphere surrounding the galaxy. When these caustic spheres are approached from the inside the density diverges as $d \sim \frac{1}{\sqrt{\sigma}}$ in the zero velocity dispersion limit. These spheres are the outer caustics in the phase-space structure of galactic halos. In addition there are inner caustics.

It is a little more difficult to see why there must be inner caustics, and to derive their structure. See refs. [3,4] for details. The inner caustics are rings. They are located near where the particles with the most angular momentum in a given in and out flow are at their distance of closest approach to the galactic center. A ring is a closed tube whose cross-section is a $D_{-4}$ catastophe ${ }^{-}$. The cross-section is shown in Fig. 2 in the limit of axial and reflection symmetry, and where the transverse dimensions, $p$ and $q$, are much smaller than the ring radius $a$. In the absence of any symmetry, the cross-section of the tube does not have the exact shape shown in Fig. 2 but it still has that shape qualitatively, i.e. it is still a closed line with three cusps one of which points away from the galactic center.

The existence of caustic rings of dark matter follows from only two assumptions:

1. the existence of collisionless dark matter

2. that the velocity dispersion of the infalling dark matter is much less, by a factor ten say, than the rotation velocity of the galaxy. 


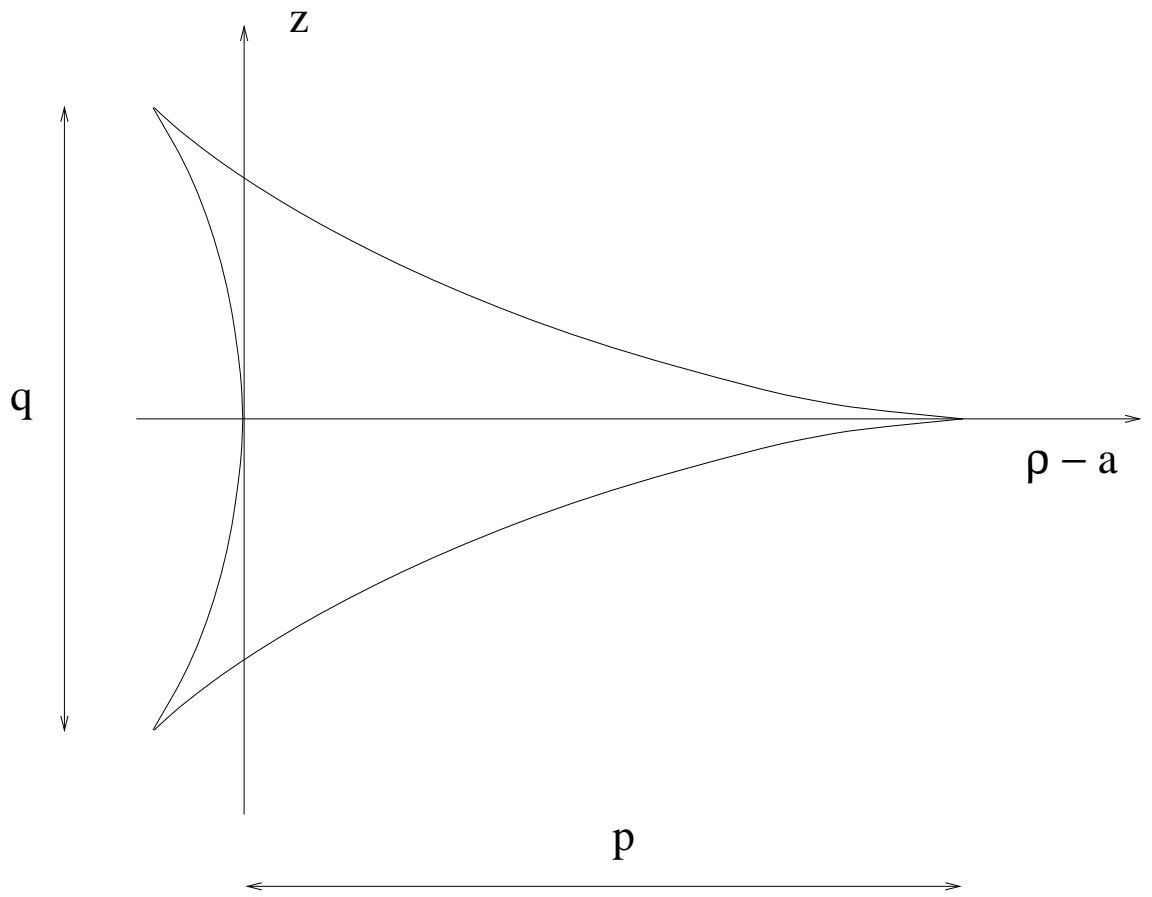

Figure 2: Cross-section of a caustic ring in the case of axial and reflection symmetry. The galactic center is to the left of the figure. The $z$-direction is orthogonal to the galactic plane. The $\rho$-direction is radial. $a$ is the caustic ring radius. The closed line with three cusps is the location of a caustic surface. The density diverges when the surface is approached from the inside as $\sigma^{-1 / 2}$ where $\sigma$ is the distance to the surface.

Only the second assumption requires elaboration. Velocity dispersion has the effect of smoothing out caustics. The question is when is the velocity dispersion so large as to smooth caustic rings over distance scales of order the ring radius $a$, thus making the notion of caustic ring meaningless. In ref. [3] this critical velocity dispersion was estimated to be $30 \mathrm{~km} / \mathrm{s}=10^{-4}$ for the caustic rings in our own galaxy, whose rotation velocity is $220 \mathrm{~km} / \mathrm{s} .10^{-4}$ is much less than the primordial velocity dispersion $\delta v$ of the cold dark matter candidates. However the velocity dispersion $\Delta v$ associated with density perturbations also smoothes caustics in coarse grained observations. So the question is whether the velocity dispersion $\Delta v$ of cold dark matter particles associated with density perturbations falling onto our galaxy is less than $30 \mathrm{~km} / \mathrm{s}$. The answer is yes with high probability since the infalling dark matter particles are not 
associated with any observed inhomogeneities. $30 \mathrm{~km} / \mathrm{s}$ is of order the velocity dispersion of the Magellanic Clouds. For the velocity dispersion of the dark matter particles presently falling onto our galaxy to be as large $30 \mathrm{~km} / \mathrm{s}$, these particles would have to be part of clumps whose mass/size ratio is of order that of the Magellanic Clouds. But if that were the case, why did these clumps fail to become luminous?

One might ask whether caustic rings can be seen in N-body simulations of galaxy formation. The generic surface_caustics associated with simple folds of the phase-space sheet have been seen 6 . However, caustic rings would require far greater resolution than presently available, at least in a $3 \mathrm{D}$ simulation of our own halo. Indeed, the largest ring in our galaxy has been estimated 3 to have radius of order $40 \mathrm{kpc}$. It is part of an in and out flow that extends to the Galaxy's current turnaround radius, of order $2 \mathrm{Mpc}$. To resolve this first ring, the spatial resolution would have to be considerably smaller than $10 \mathrm{kpc}$. Hence a minimum of $2 \cdot \frac{1}{(10 \mathrm{kpc})^{3}} \frac{4 \pi}{3}(2 \mathrm{Mpc})^{3} \simeq 7 \cdot 10^{7}$ particles would be required to see the caustic ring in a simulation of this one flow. However, the number of flows at $40 \mathrm{kpc}$ in our halo $\mathrm{B}$ is of order 10 . So it appears that $10^{9}$ particles is a strict minimum in a $3 \mathrm{D}$ simulation of our halo. Even so, this addresses only the kinematic requirement of resolving the halo in phase-space, assuming moreover that the particles are approximately uniformly distributed on the phase-space sheets. There is a further dynamical requirement that 2-body collisions do not artificially 'fuzz up' the phase-space sheets. Indeed 2-body collisions are entirely negligible in the flow of cold dark matter particles such as axions or WIMPs. On the other hand, 2-body collisions are present, and hence the velocity dispersion is artificially increased, in the simulations. This may occur to such an extent that the caustics are washed away even if $10^{9}$ particles are used.

In the self-similar infall model明 of galactic halo formation the caustic ring radii $a_{n}$ are predicted

$$
\left\{a_{n}: n=1,2, \ldots\right\} \simeq(39,19.5,13,10,8, \ldots) \mathrm{kpc}\left(\frac{j_{\max }}{0.25}\right)\left(\frac{0.7}{h}\right)\left(\frac{v_{r o t}}{220 \frac{k m}{s}}\right)
$$

where $h$ is the present Hubble rate in units of $100 \mathrm{~km} / \mathrm{s} . \mathrm{Mpc}, v_{\text {rot }}$ is the rotation velocity of the galaxy and $j_{\max }$ is the maximum of its dimensionless angular momentum distribution as defined in ref. [8]. In Eq. (1) we assume that the parameter畞 $\epsilon=0.3$.

Eq. (11) predicts the caustic ring radii of a galaxy in terms of its first ring radius $a_{1}$. If the caustic rings lie close to the galactic plane they cause bumps in the rotation curve, at the caustic ring radii. As a possible example of this 
effect, consider 3 the rotation curve of NGC3198, one of the best measured. It has three faint bumps at radii: $28,13.5$ and $9 \mathrm{kpc}$, assuming $h=0.75$. The ratios happen to be consistent with Eq. (11) assuming the bumps are caused by the first three $(n=1,2,3)$ ring caustics of NGC3198. Moreover, since $v_{\text {rot }}=150 \mathrm{~km} / \mathrm{s}, j_{\max }$ is determined to equal 0.28 . The uncertainty in $h$ is a systematic effect that can be corrected for when determining $j_{\max }$ because the bump radii scale like $1 / h^{\prime}$ where $h^{\prime}$ is the Hubble rate assumed by the observer in constructing the rotation curve, and the caustic ring radii scale as $1 / h$. Rises in the inner rotation curve of the Milky Way were also interpreted 3 as due to caustics $n=6,7,8,9,10,11,12$ and 13 . This determined the value of $j_{\max }$ of our own galaxy to be 0.263 . The first five caustic ring radii in our galaxy are then predicted to be: $41,20,13.3,10,8 \mathrm{kpc}$.

\section{Evidence for universal structure in galactic halos}

Motivated by these findings, we analyzed 9 a set of 32 extended well-measured galactic rotation curves which had been previously selected 10 under the criteria that each is an accurate tracer of the galactic radial force law, and that it extends far beyond the edge of the luminous disk.

According to the self-similar caustic ring model, each galaxy has its own value of $j_{\max }$. Over the set of 32 galaxies selected in ref. [10], $j_{\max }$ has some unknown distribution. However, the fact that the values of $j_{\max }$ of NGC3198 and of the Milky Way happen to be close to one another, within 7\%, suggests that the $j_{\max }$ distribution may be peaked near a value of 0.27 . Our strategy is to rescale each rotation curve according to

$$
r \rightarrow \tilde{r}=r\left(\frac{220 \mathrm{~km} / \mathrm{s}}{v_{\text {rot }}}\right)
$$

and to add them in some way. Since Eq. (11) predicts the $n$th caustic radius $a_{n}$ to be distributed like $j_{\max }$ for all $\mathrm{n}$, and it fixes the ratios $a_{n} / a_{1} \simeq 1 / n$, the sum of rotation curves should show the $j_{\max }$ distribution, once for $n=1$, then at about half the $n=1$ radii for $n=2$, then at about $1 / 3$ the $n=1$ radii for $n=3$,and so on. If the $j_{\max }$ distribution is broad, the sum of rotation curves is unlikely to show any feature. However, if it is peaked, then the sum should show a peak for $n=1$ at some radius, then again at $1 / 2$ that radius for $n=2$, at $1 / 3$ the radius for $n=3$, and so on. If the $j_{\max }$ distribution is peaked at 0.263 (the value for the Milky Way) the peaks in the sum of rotation curves should appear at $41 \mathrm{kpc}, 20 \mathrm{kpc}, 13.3 \mathrm{kpc} \ldots$

The procedure followed to add the 32 rotation curves is described in detail in ref. [9]. Briefly, we proceeded as follows. For each rotation curve, all data 


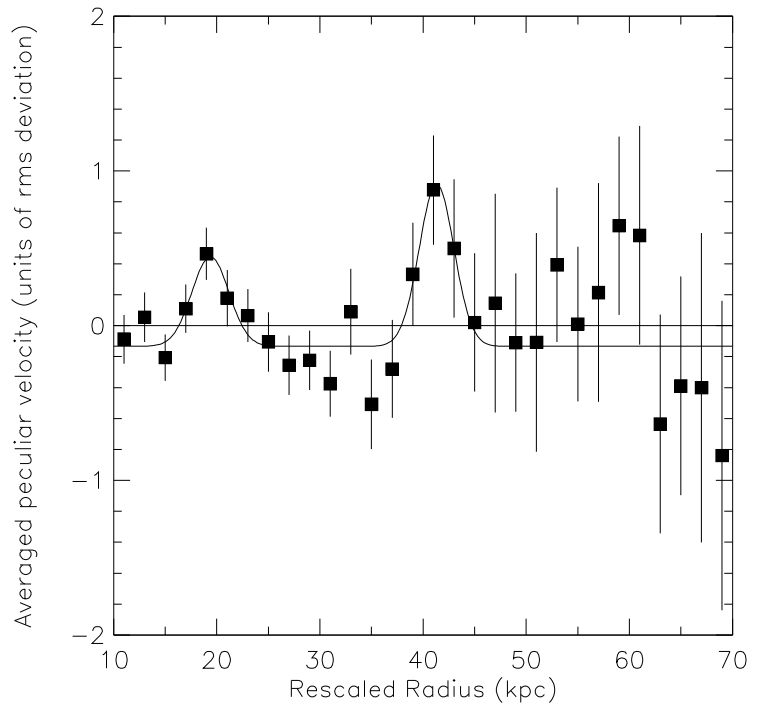

Figure 3: Binned data for 32 galaxy sample, with peaks fit to Gaussians.

points with rescaled radii $\tilde{r}<10 \mathrm{kpc}$ were deleted to remove the effect of the luminous disk. The remaining points were then fitted to a line. The rotation velocity $v_{\text {rot }}$ used to rescale the radii in Eq. (2) is the average of that line. The rms deviation $\sqrt{\left\langle\delta v^{2}\right\rangle}$ from the linear fit was determined for each galaxy. This was taken to be the error on the residuals $\delta v_{i}$, i.e. the differences between the measured velocities in a rotation curve and the linear fit. Finally the sample of 32 galaxies was averaged in $2 \mathrm{kpc}$ radial bins:

$$
b_{i} \equiv \frac{1}{N_{i}} \sum_{j=1}^{N_{i}} \delta \tilde{v}_{j}
$$

where $N_{i}$ is the number of data points in the bin. The assigned error on each $b_{i}$ is then simply $1 / \sqrt{N_{i}}$. Fig. 3 shows the result.

There are two features evident at roughly 20 and $40 \mathrm{kpc}$. A fit to two Gaussians plus a constant indicates features at $19.4 \pm 0.7 \mathrm{kpc}$ and $41.3 \pm 0.8 \mathrm{kpc}$, with overall significance of $2.4 \sigma$ and $2.6 \sigma$, respectively. Fig. 3 shows the fitted 
curve. When the same fit is applied to the same data in $1 \mathrm{kpc}$ bins, the significance of the two peaks is 2.6 and $3.0 \sigma$ respectively. The locations of the features agrees with the predictions of the self-similar caustic ring model with the $j_{\max }$ distribution peaked at 0.27 . The use of Gaussians to fit the peaks in the combined rotation curve was an arbitrary choice in the absence of information on the $j_{\max }$ distribution.

The existence of velocity peaks and caustic rings in the cold dark matter

distribution is relevant to axion 11 and WIMP searches 12. Caustics may also

be investigated using gravitational lensing techniques 13.

\section{Acknowledgements:}

This work was supported in part by the US Department of Energy under grant No. DEFG05-86ER40272.

\section{References}

1. S. Tremaine and J.E. Gunn, Phys. Rev. Lett. 42407 (1979); J.R. Bond, G. Efstathiou and J. Silk, Phys. Rev. Lett. 451980 (1980); S.D.M. White, C.S. Frenk and M. Davis, Ap. J. 274 L1 (1983).

2. J.R. Ipser and P. Sikivie, Phys. Lett. B291 288 (1992).

3. P. Sikivie, Phys. Lett. B. 432139 (1998).

4. P. Sikivie, Phys. Rev. D60 063501 (1999).

5. R. Gilmore, Catastrophy Theory for Scientists and Engineers, Wiley, 1981.

6. A.G. Doroshkevich et al., M.N.R.A.S. 192321 (1980); A.A. Klypin and S.F. Shandarin, M.N.R.A.S. 204891 (1983); J.M. Centrella and A.L. Melott, Nature 305196 (1983); A.L. Melott and S.F. Shandarin, Nature 346633 (1990).

7. J. A. Filmore and P. Goldreich, Ap. J. 2811 (1984); E. Bertschinger, Ap. J. Suppl. 5839 (1985).

8. P. Sikivie, I.I. Tkachev and Y. Wang, Phys. Rev. Lett. 752911 (1995) and Phys. Rev. D56 1863 (1997).

9. W. Kinney and P. Sikivie, Phys. Rev. D, 61:087305 (2000).

10. K. G. Begeman, A. H. Broeils and R. H. Sanders, MNRAS 249523 (1991); R. H. Sanders, Ap. J. 473117 (1996).

11. P. Sikivie, astro-ph/9810286, published in The Identification of Dark Matter, edited by N. Spooner and V. Kudryavtsev, World Scientific 1999, pp. 58-65,

12. C. Copi, J. Heo and L. Krauss, Phys. Lett. B461 43 (1999).

13. C. Hogan, Ap. J. 52742 (1999). 